\title{
Acyclic nucleoside phosphonates: a new dimension to the chemotherapy of DNA virus and retrovirus infections
}

\begin{abstract}
Most antiviral compounds that are currently used in the treatment of infection with herpes simplex virus (HSV), varicella-zoster virus (VZV) and cytomegalovirus (CMV) are acyclic nucleoside analogues: viz., acyclovir, penciclovir and ganciclovir [1]. To increase their oral bioavailability, acyclovir and penciclovir have been converted to their oral prodrug forms, valaciclovir and famciclovir, respectively.
\end{abstract}

After uptake by cells, these compounds are phosphorylated through three consecutive phosphorylation steps before they interact, in their triphosphate form, with their target enzyme, the viral DNA polymerase. The first phosphorylation step is accomplished by a specific virus-encoded thymidine kinase (TK) (for HSV and VZV) or a specific virus-encoded protein kinase (PK) (for CMV), after which cellular kinases complete the phosphorylation process. In their triphosphate form, the compounds then act as competitive inhibitors or alternative substrates for the DNA polymerase, and, if incorporated into the DNA chain, they may act as chain terminators, thus preventing further chain elongation. In addition, ganciclovir and penciclovir may be incorporated, via an internucleotide linkage, in the interior of the DNA chain.

The first phosphorylation step is crucial for the antiviral activity of the acyclic nucleoside analogues. It excludes activity of the compounds against those viruses that either do not induce a specific TK or PK or have developed resistance to the compounds through mutations in these enzymes [2]. Thus, acyclovir, penciclovir and ganciclovir are ineffective against $\mathrm{TK}^{-} \mathrm{HSV}, \mathrm{TK}^{-} \mathrm{VZV}, \mathrm{PK}^{-} \mathrm{CMV}$ and any other DNA viruses (i.e., polyoma-, papilloma-, adeno- and poxviruses) that fail to secure phosphorylation of the nucleoside to the nucleoside monophosphate.

Acyclic nucleoside phosphonates [3] possess a phosphonate group attached to the acyclic nucleoside moiety through a stable $\mathrm{P}-\mathrm{C}$ bond. In contrast to the phosphate group (which is attached through a $\mathrm{P}$ $\mathrm{O}-\mathrm{C}$ bond), a phosphonate group cannot be cleaved by cellular hydrolases. Foremost among the acyclic nucleoside phosphonates that have been pursued as antiviral agents are cidofovir, adefovir and (R)-9-(2phosphonylmethoxypropyladenine) (PMPA). Because of their limited oral bioavailability, the latter two compounds have been converted to oral prodrug forms: bis(POM)PMEA (adefovir dipivoxil) and bis (POC)PMPA.

As the acyclic nucleoside phosphonates already contain a phosphate-mimetic group, they need only two, instead of three, phosphorylation steps to reach the active metabolite stage. In 'by-passing' the primary nucleoside kinase step, the acyclic nucleoside phosphonates may be expected to act against a broad range of DNA viruses, including hepatitis B virus (HBV) and retroviruses like human immunodeficiency virus (HIV); i.e., those viruses with a DNA polymerase that allows the active metabolites of the acyclic nucleoside phosphonates to compete with the normal substrates.

Cidofovir is phosphorylated by pyrimidine nucleoside monophosphate kinase to cidofovir monophosphate, which is then further phosphorylated by nucleoside diphosphate kinase, pyruvate kinase or creatine kinase to cidofovir diphosphate [4]. Both phosphorylation steps can occur in both uninfected and virus-infected cells, but are accentuated in the virus-infected cells. For adefovir, and presumably PMPA, phosphorylation to the diphosphate form can be achieved in one or two steps through the aid of 5-phosphoribosyl-1-pyrophosphate synthetase [5], or AMP (dAMP) kinase [6], respectively.

The acyclic nucleoside phosphonates owe their selective antiviral activity to the fact that, in their diphosphorylated form, they have a higher affinity (lower $\mathrm{K}_{\mathrm{i}}$ value) for the viral DNA polymerases than for the corresponding cellular enzymes $[7,8]$. In this form they act as competitive inhibitors or alternative substrates for the viral DNA polymerases. The incorporation of one molecule of adefovir or PMPA at the $3^{\prime}$ end of the growing DNA chain suffices to terminate further chain elongation [7]. PMPA is a more faithful chain terminator of the HIV-1 reverse transcriptase reaction than adefovir, as it is incorporated to a lower extent by the cellular DNA polymerases [9]. For cidofovir two consecutive incorporations are required to halt DNA elongation efficiently [10].

Cidofovir $[11,12]$ is active against all herpesviruses, 
including Epstein-Barr virus (EBV), human herpes virus type 6 (HHV-6), human herpes virus type 7 (HHV-7) and human herpes virus type 8 (HHV-8, the putative cause of Kaposi's sarcoma) [13] as well as thymidine kinase-deficient strains of HSV and VZV, and protein kinase-deficient strains of $\mathrm{CMV}$; it is also active against adeno-, polyoma-, papilloma- and poxviruses [14-17].

The antiviral activity of adefovir is unique in that it encompasses both retroviruses and hepadnaviruses as well as herpesviruses [7]. This means that it could be used for the treatment of HIV and HBV infections as well as for the prophylaxis (or therapy) of herpesvirus (particularly CMV) infections. In contrast, the activity of PMPA is confined to retroviruses and hepadnaviruses. Both adefovir and PMPA are active against simian immunodeficiency virus (SIV), feline immunodeficiency virus (FIV), visna-maedi virus and murine leukaemia/sarcoma viruses [7]. Particularly striking are the effects that have been recorded for PMPA in the prevention of SIV infection in adult macaques [18] and the treatment of chronic SIV infections in infant rhesus macaques [19] and adult cynomolgus macaques [20].

The acyclic nucleoside phosphonates offer great promise for the treatment of a large variety of DNA virus and retrovirus infections. Cidofovir delays progression of CMV retinitis in patients with AIDS $[21,22]$, and is approved for this indication in the USA and Europe. It has to be given intravenously at $5 \mathrm{mg} / \mathrm{kg}$ once weekly for 2 weeks and then once every other week, a regimen that is associated with manageable side effects. Monitoring of renal function before the initiation of cidofovir therapy and concomitant administration of probenecid and saline hydration are required to minimise drug-related nephrotoxicity $[21,22]$.

There are a vast number of indications for which cidofovir could be further pursued. These include, besides CMV retinitis, HSV, VZV and CMV infections that have become refractory to acyclovir, penciclovir or ganciclovir treatment, EBV, HHV-6, HHV-7 and HHV-8 infections (i.e., Kaposi's sarcoma), adenovirus infections (i.e., keratoconjunctivitis), poxvirus infections (i.e., molluscum contagiosum), polyomavirus infections (such as progressive multifocal leukoencephalopathy), and various papillomavirus infections, such as anogenital warts, recurrent laryngeal papillomas and cervical intra-epithelial neoplasias [12]. Complete and permanent remissions of papillomatous lesions have been achieved following local intralesional injections of cidofovir: an hypopharyngeal-oesophageal papilloma, due to human papilloma virus type 16 , showed complete regression after topical cidofovir therapy [23].

The oral prodrug forms of adefovir and PMPA are under clinical trial for the treatment of HIV infections. The suitability of adefovir dipivoxil for the treatment of chronic HBV infections is also under study. PMPA is being explored as a vaginal microbicide in the prevention of sexual transmission of HIV.

The main advantage of the acyclic nucleoside phosphonates over the 'classical' nucleoside analogues is their prolonged antiviral action, which allows infrequent dosing, i.e., once daily for the oral formulations of adefovir and PMPA; once weekly (or once every 2 weeks) for intravenous or intralesional cidofovir; once daily (for a few days) for cidofovir if applied topically as gel or eyedrops. Such regimens offer much greater convenience and encourage better compliance from the patients.

Inevitably, resistance to acyclic nucleoside phosphonates is likely to arise by mutations in the target enzyme (i.e., the herpesvirus DNA polymerase for cidofovir and the HIV reverse transcriptase for adefovir and PMPA). Such resistance is well-known for the reverse transcriptase inhibitors and protease inhibitors that are being used for the treatment of HIV infections [24]. Yet, although cidofovir-resistant CMV strains and adefovir-resistant HIV-1 strains have been found in vitro after prolonged exposure of the viruses to the drugs, neither compound (nor PMPA) have so far been shown to select mutant strains that may compromise their clinical efficacy in vivo $[25,26]$.

Acyclic nucleoside phosphonates offer attractive prospects for the treatment of DNA virus and retrovirus infections: they are active against viruses such as polyoma-, adeno-, papilloma- and poxviruses for which there is no current antiviral chemotherapy; they are active against herpesvirus infections which are either not amenable to other antiviral agents or have become resistant to these antiviral agents (such as variants that are resistant to acyclovir, penciclovir and ganciclovir); they show combined inhibitory effects on both retrovirus (i.e., $\mathrm{HIV}$ ) and DNA virus (i.e., CMV) infections that may occur simultaneously in the same patients; they provide a prolonged antiviral response lasting for several days or even longer; and they do not readily lead to the emergence of antiviral drug resistance that may compromise their clinical effectiveness.

I thank Christiane Callebaut for her proficient editorial help.

ERIK DE CLERCQ, Rega Institute for Medical Research, Katholieke Universiteit Leuven, Minderbroedersstraat 10 , B-3000 Leuven, Belgium

\section{References}

1. De Clercq E. Trends in the development of new antiviral agents for the chemotherapy of infections caused by herpesviruses and retroviruses. Rev Med Virol 1995; 5: 149-164.

2. Field AK, Biron KK. "The end of innocence" revisited: resistance of herpesviruses to antiviral drugs. Clin Microbiol Rev 1994; 7: 1-13. 
3. De Clercq E. Broad-spectrum anti-DNA virus and antiretrovirus activity of phosphonylmethoxyalkylpurines and pyrimidines. Biochem Pharmacol 1991; 42: 963-972.

4. Cihlar T, Chen MS. Identification of enzymes catalyzing twostep phosphorylation of cidofovir and the effect of cytomegalovirus infection on their activities in host cells. Mol Pharmacol 1996; 50: 1502-1510.

5. Balzarini J, Navé J-F, Becker MA, Tatibana M, De Clercq E. Kinetic properties of adenine nucleotide analogues against purified 5-phosphoribosyl-1-pyrophosphate synthetases from $E$. coli, rat liver and human erythrocytes. Nucleosides Nucleotides 1995; 14: 1861-1871.

6. Merta A, Votruba I, Jindrich J et al. Phosphorylation of 9-(2phosphonomethoxyethyl)adenine by AMP(dAMP) kinase from L1210. Biochem Pharmacol 1992; 44: 2067-2077.

7. Naesens L, Snoeck R, Andrei G, Balzarini J, Neyts J, De Clercq E. HPMPC (cidofovir), PMEA (adefovir) and related acyclic nucleoside phosphonate analogues: a review of their pharmacology and clinical potential in the treatment of viral infections. Antiviral Chem Chemother 1997; 8: 1-23.

8. Kramata P, Votruba I, Otová B, Holý A. Different inhibitory potencies of acyclic phosphonomethoxyalkyl nucleotide analogs toward DNA polymerases $\alpha, \lambda$ and $\epsilon$. Mol Pharmacol 1996; 49: 1005-1011.

9. Cihlar T, Chen MS. Incorporation of selected nucleoside phosphonates and anti-human immunodeficiency virus nucleotide analogues into DNA by human DNA polymerases $\alpha, \beta$ and $\gamma$. Antiviral Chem Chemother 1997; 8: 187-195.

10. Xiong X, Smith JL, Chen MS. Effect of incorporation of cidofovir into DNA by human cytomegalovirus DNA polymerase on DNA elongation. Antimicrob Agents Chemother 1997; 41: 594-599.

11. De Clercq E. Therapeutic potential of HPMPC as an antiviral drug. Rev Med Virol 1993; 3: 85-96.

12. De Clercq E. Therapeutic potential of cidofovir (HPMPC, Vistide $^{\mathrm{TM}}$ ) for the treatment of DNA virus (i.e. herpes-, papova-, pox- and adenovirus) infections. Verh $K$ Acad Geneeskd Belg 1996; 58: 19-49.

13. Kedes DH, Ganem D. Sensitivity of Kaposi's sarcomaassociated herpesvirus replication to antiviral drugs. $J$ Clin Invest 1997; 99: 2082-2086.

14. Reymen D, Naesens L, Balzarini J, Holy A, Dvoráková H, De Clercq E. Antiviral activity of selected acyclic nucleoside analogues against human herpesvirus 6. Antiviral Res 1995; 28: $343-357$.
15. Takahashi K, Suzuki M, Iwata Y, Shigeta S, Yamanishi K, De Clercq E. Selective activity of various nucleoside and nucleotide analogues against human herpesvirus 6 and 7 . Antiviral Chem Chemother 1997; 8: 24-31.

16. Andrei G, Snoeck R, Vandeputte M, De Clercq E. Activities of various compounds against murine and primate polyomaviruses. Antimicrob Agents Chemother 1997; 41: 587-593.

17. de Oliveira CBR, Stevenson D, LaBree L, McDonnell PJ, Trousdale MD. Evaluation of cidofovir (HPMPC, GS-504) against adenovirus type 5 infection in vitro and in a New Zealand rabbit ocular model. Antiviral Res 1996; 31: 165-172.

18. Tsai C-C, Follis KE, Sabo A et al. Prevention of SIV infection in macaques by $(R)$-9-(2-phosphonylmethoxypropyl)adenine. Science 1995; 270: 1197-1199.

19. Van Rompay KKA, Cherrington JM, Marthas ML et al. 9-[2(phosphonomethoxy)propyl]adenine therapy of established simian immunodeficiency virus infection in infant rhesus macaques. Antimicrob Agents Chemother 1996; 40: 2586-2591.

20. Tsai C-C, Follis KE, Beck TW, Sabo A, Bischofberger N, Dailey PJ. Effects of (R)-9-(2-phosphonylmethoxypropyl)adenine monotherapy on chronic SIV infection in macaques. AIDS Res Hum Retroviruses 1997; 13: 707-712.

21. Lalezari JP, Stagg RJ, Kuppermann $\mathrm{BD}$ et al. Intravenous cidofovir for peripheral cytomegalovirus retinitis in patients with AIDS. Ann Intern Med 1997; 126: 257-263.

22. Studies of Ocular Complications of AIDS Research Group in Collaboration with the AIDS Clinical Trials Group. Parenteral cidofovir for cytomegalovirus retinitis in patients with AIDS: the HPMPC peripheral cytomegalovirus retinitis trial. Ann Intern Med 1997; 126: 264-274.

23. Van Cutsem E, Snoeck R, Van Ranst $M$ et al. Successful treatment of a squamous papilloma of the hypopharynxesophagus by local injections of (S)-1-(3-hydroxy-2-phosphonylmethoxypropyl)cytosine. $J$ Med Virol 1995; 45: 230 235.

24. De Clercq E. Development of resistance of human immunodeficiency virus (HIV) to anti-HIV agents: how to prevent the problem? Int J Antimicrob Agents 1997, 9: 21-36.

25. Cherrington JM, Mulato AS, Fuller MD, Chen MS. Novel mutation $(\mathrm{K} 70 \mathrm{E})$ in human immunodeficiency virus type 1 reverse transcriptase confers decreased susceptibility to 9-[2(phosphonomethoxy)ethyl]adenine in vitro. Antimicrob Agents Chemother 1996; 40: 2212-2216.

26. Cherrington JM. Human cytomegalovirus: resistance profile of cidofovir. Int Antiviral News 1997; 5: 91-92. 